\title{
Marguerite Duras ou le mélange des genres
}

\author{
Şengül Kocaman \\ Université Dicle
}

Au début du mois d'août 2014, le monde entier apprenait le décès par suicide de l'acteur américain Robin Williams, qui aurait souffert de dépression. Dans ces moments-là, les langues se délient : ceux qui l'ont approché ou qui croyaient le connaître se sont mis à concéder que sa situation s'était dégradée depuis quelque temps, lorsqu'il a dû se contenter de seconds rôles au cinéma. Le problème est que Robin Williams n'est pas la première personnalité connue à connaître un déclin précoce de son vivant; d'autres vedettes du cinéma, de la chanson, du 
monde des arts, ont aussi connu la disgrâce. Et quand on voit ce qu'il advient des vivants, comment en irait-il autrement de ceux qui ont disparu physiquement? Combien de grands artistes, adulés de leur vivant, on échappé à la disgrâce sitôt morts?

Marguerite Duras est décédée en 1996. Née en 1914, elle aurait eu cent ans en 2014, ce qui a fourni à diverses institutions l'occasion de commémorer ce centenaire. C'est aussi, d'un certain point de vue, l'occasion de tester sur le public ce qui reste de notoriété pour cet auteur qui connut son heure de gloire moins d'une quinzaine d'années avant sa mort, avec l'adaptation cinématographique de son roman L'Amant. L'idée de cet article nous est venue du rapprochement avec un autre écrivain qui connut aussi son heure de gloire, Eugène Ionesco, entré de son vivant dans la prestigieuse collection de la Bibliothèque de la Pléiade, mais admis également à l'Académie française. Il se trouve que, vingt ans après sa mort, le dramaturge Ionesco n'apparaît plus que fort rarement au fronton de grands théâtres parisiens, voire français. En ce qui la concerne, de prime abord, il nous a semblé qu'indépendamment des manifestations organisées par des grandes institutions, à l'instar de la BNF (Bibliothèque nationale de France), c'est surtout la Duras auteur de pièces de théâtre qui était célébrée par les médias et par le public, seul décisionnaire en matière de notoriété d'un artiste. Mais en suggérant cela, nous n'avons pas l'impression d'avoir énormément fait avancer la réflexion, car la définition même de pièce de théâtre reste problématique chez cet auteur. 


\section{Hommage avant tout à l'auteur de théâtre}

À vrai dire, notre intérêt pour le «cas Duras » a été suscité par quelques coupures de presse, comme cette présentation d'une « année Duras » par un quotidien français :

2014, année Duras : on fête les cent ans de la naissance de cette immense romancière, qui a aussi écrit pour le théâtre... Quatre pièces d'envergure sont actuellement à l'affiche à Paris.

C'est le moment ou jamais de (re)découvrir le théâtre de Marguerite Duras. Voici le programme des réjouissances en ce début d'années sur les scènes parisiennes.

$[\ldots]$

La plus intime

Au théâtre du Vieux Colombier, Muriel Mayette, directrice de la Comédie-Française, met en scène La Maladie de la mort, un long poème en prose, écrit en 1982, au moment où Duras est au plus mal, victime d'une cirrhose aiguë doublée d'une grave dépression.

\section{$[\ldots]$}

La plus œdipienne

Fanny Ardant, Nicolas Duvauchelle et Agathe Bonitzer sont réunis par Thierry Klifa au théâtre de la Gaîté-Montparnasse, pour jouer Des journées entières dans les arbres. Avant de devenir pièce, le texte fut une nouvelle [...].

La plus culte

Savannah Bay est une pièce bouleversante écrite en 1982 et entrée au répertoire de la Comédie-Française en 2002. C'est au théâtre de l'Atelier qu'elle se joue, mise en scène par Didier Bezace. La grande Emmanuelle Riva (l'héroïne d'Hiroshima mon amour, mais aussi d'Amour de Haneke) revient aux origines en jouant Duras. À ses côtés, la non moins talentueuse Anne Consigny. Didier Bezace monte également à l'Atelier deux autres pièces de Duras : Le Square et Marguerite et le président. 
$[\cdots]$

La plus novatrice

Au Théâtre de Belleville, on ne joue pas une pièce de Duras mais sur Duras: Marguerite et moi. Fatima Soualhia Manet \& Christophe Casamance nous font découvrir au travers d'extraits d'interviews et d'entretiens issus de grands moments de radio ou de télévision, l'incroyable personnalité de l'écrivain et son regard sur le monde. (Gandillot)

Il est question d'une "immense romancière qui a aussi écrit pour le théâtre », et ce sont précisément des pièces de théâtre qui vont jalonner l'année de commémoration, en tout cas, susciter l'intérêt des médias. Un certain nombre de grands organes de presse sont venus conforter cette impression :

Marguerite Duras a bousculé beaucoup de conventions de genres en matière de création artistique. Elle a imprimé son regard singulier et son expérience personnelle sur pellicule ou sur papier, laissant ainsi son nom dans l'Histoire.

En ce début d'année 2014, les programmations théâtrales célèbrent le centenaire de la naissance de cette femme puissante et fragile à la fois, en proposant à l'affiche quelquesunes de ses pièces phares ainsi que des entretiens de l'écrivain.

Savannah Bay, Le Square, Des journées entières dans les arbres, La Maladie de la mort, L'Homme atlantique, Hiroshima mon amour, Un barrage contre le Pacifique... Rendez-vous au Théâtre de l'Atelier, à la Gaîté Montparnasse, au Théâtre de l'Athénée, au Théâtre de Belleville, au Lucernaire ou encore aux Artistic Athévains pour réentendre sur scène l'écriture et la parole intransigeantes de cette femme de lettres pleine de tempérament, de blessures et de failles, dont la plume était, avant toute chose, irriguée par sa vie et ses amours. (Plantin)

Le 20 janvier 2014, à la veille de présenter la pièce Des journées entières dans les arbres au théâtre parisien de la GaîtéMontparnasse, la comédienne Fanny Ardant était reçue sur le plateau du journal télévisé de 13 heures de France 2, ce qui a dû 
assurer une excellente promotion à la pièce. Dans sa version mise en ligne sur Internet et réactualisée quelques semaines plus tard, l'interview est précédée d'un reportage commençant par un plan sur de longues files d'attente devant le théâtre :

Chaque soir devant le théâtre, la file d'attente est impressionnante, mais c'est toujours comme ça quand Fanny Ardant est à l'affiche. Pour son retour sur les planches, la comédienne a choisi une mère inventée par Marguerite Duras en 1954. Une mère aussi extravagante que tendrement dévastatrice.

[Thierry Klifa : C'est un rôle qui aurait pu être écrit pour Fanny Ardant; elle en a la démesure, la folie, le côté passionnel, et Fanny, elle n'a pas peur d'aller très loin.]

Et heureusement qu'en scène elle n'a peur de rien, Fanny Ardant, car ce rôle de mère, qui vient déclarer son amour exclusif à son vaurien de fils, requiert force, fantaisie, folie, douleur aussi.

[F. A. : On choisit toujours ce qu'on aime; pour moi, la vie est trop courte pour aller vers un personnage qui m'ennuierait prodigieusement. Rester une heure et demie sur scène et ne pas revendiquer absolument tout ce que dirait cette femme, non!]

Si Marguerite Duras était encore en vie, elle aurait dit de Fanny Ardant dans ce rôle: "sublime, forcément sublime.» (Belhaoues)

Q. Pour ceux qui ne connaissent pas encore cette pièce, qui est une pièce très très forte, le point de départ, ce sont en fait les retrouvailles entre une mère et son fils, ce fils qu'elle n'a pas vu depuis cinq ans.

R. C'est le fils préféré d'une famille de cinq enfants et c'est l'amour déraisonnable d'une mère pour son fils qu'elle a toujours gardé dans l'enfance et qu'elle a laissé des journées entières dans les arbres, plutôt que de l'élever pour faire un bon citoyen, et l'école, et les examens, et les mariages et les situations. Et c'est une heure et demie, c'est le dernier voyage parce que cette femme va mourir, de tenter de dire à son fils : 
viens avec moi, et en même temps les aveux, et en même temps, comme dans Qui a peur de Virginia Woolf?, là c'est un couple d'amoureux tandis qu'ici, c'est un couple d'amour fou et de haine destructrice, des reproches, des aveux, des déclarations.

Q. Si je dis que c'est une mère dévorante d'amour, est-ce que c'est ça?

R. Oui. Dévorante parce que d'amour fou. Toute la société passe son temps à nous dire qu'il faut être raisonnable, faire les choses avec parcimonie, économie, et elle, c'est tout le contraire... Elle le dit: «je suis contradictoire, mais je m'y retrouve ", et elle ne peut pas s'en empêcher; c'est plus fort qu'elle.

Q. C'est volcanique, c'est primitif, puisque c'est plus fort qu'elle; est-ce qu'on est dans la démesure, est-ce qu'on est dans la folie?

R. Il y a toujours ce moment où justement quand on lâche les amarres, il n'y a plus ce sujet, verbe, complément...

Q. Il n'y a plus de cadre...

Il n'y a plus de cadre; il y a une sorte de démesure de la confession.

[Extrait du spectacle]

Q. Ce texte est tellement subversif qu'il a été censuré. On a du mal à l'imaginer aujourd'hui.

R. Elle a d'abord commencé à écrire la nouvelle, puis elle a écrit cette pièce, dans les années soixante... Parce que je crois que c'est subversif par rapport à tout, pas simplement pour la relation entre la mère et le fils mais aussi pour ses déclarations; c'est comme une déclaration de guerre contre la société.

Q. C'est presque libertaire.

R. Oui, elle dit : les gens qui travaillent me dégoûtent. Parce que ce fils qui est devenu un peu un gigolo, qui va dans les boîtes de nuit, qui vit d'expédients, elle le traite comme un prince, c'est-àdire que tout ce qui serait laborieux, bien-pensant, c'est pour ça que c'est dangereux pour une société. 
Q. Pour vous, dans cette pièce, il y a une première rencontre, celle avec Nicolas Duvauchelle, et une autre, qui n'en est pas une puisque vous adorez les mots de Duras, que vous avez déjà joué plusieurs fois du Duras: La Maladie de la mort, La Bête dans la jungle avec Depardieu. Donc il y a à la fois une première rencontre et puis cet amour avec les mots de Duras.

R. Moi, j'ai tendance à être un peu comme la mère: je suis exagérément passionnelle. Donc j'ai toujours aimé Marguerite Duras, et pour une actrice, dire ces mots, c'est comme pour une pianiste jouer de la musique ou pour une violoncelliste. Les mots amènent l'émotion, la scansion de la phrase, du rythme, apportent quelque chose. Une vraie partition.

Q. Merci à vous Fanny Ardant; c'est une pièce que tout le monde attend, il faut le dire; on a très envie de la voir... et ça commence demain... (Lucet)

Lors de l'interview qui précède, on ne sait plus très bien si la journaliste s'enflamme pour une pièce qu'elle aurait déjà vue («c'est volcanique, c'est primitif, puisque c'est plus fort qu'elle...»), et que sa consœur, citée plus haut, trouvait déjà sublime, ou si c'est juste une pièce qu'elle brûle d'envie de découvrir (" c'est une pièce que tout le monde attend, il faut le dire; on a très envie de la voir... et ça commence demain »).

Il ressort en tout cas de ce qui précède une forte impression d'enthousiasme, voire de dithyrambe, que nous retrouvons dans un autre organe de presse, qui parle « d'ensemble très beau, harmonieux..., d'événement» :

$\mathrm{Au}$ Théâtre de l'Atelier, trois spectacles sont à l'affiche: Marguerite et le président, d'après le célèbre entretien publié par L'Autre Journal, avec Jean-Marie Galey et une petite fille, Le Square avec Clotilde Mollet et le metteur en scène lui-même, Savannah Bay qui voit le retour à la scène d'Emmanuelle Riva et d'Anne Consigny. Remarquable.

Le dimanche, on peut voir les trois spectacles et l'on ne saurait trop vous recommander de choisir cette solution car l'ensemble 
est vraiment très beau, harmonieux et donné sur d'excellents rythmes. De plus chacun des spectacles n'excède pas une heure quinze et l'on ne voit pas le temps passer.

Un décor unique à dominante claire et simplement transformé : une table pour Marguerite et le président, un amoncellement de chaises pour Le Square, un ponton pour Savannah Bay. [...]

Enfin vient Savannah Bay. Un événement puisque cette production voit le retour d'Emmanuelle Riva qui n'avait plus joué au théâtre depuis Médée, dans la mise en scène de Jacques Lassalle, avec Isabelle Huppert dans le rôle-titre.

Laura Pels, directrice du Théâtre de l'Atelier, lui a proposé de jouer la femme "dans la splendeur de l'âge » imaginée par Marguerite Duras. Après Amour de Haneke, que peut-on désirer? Elle a dit oui. Elle a pensé à Anne Consigny. Elles ont pensé à Didier Bezace.

Et cela donne une version exceptionnelle de la pièce. Elle a deux versions. Didier Bezace est revenu en partie à la première. Douleur insondable, amour. Vie, sensualité, sensibilité. (Héliot)

« Il y a là un sommet de l'art de l'interprétation, un sommet d'intelligence, de musique, de délicatesse. On comprend complètement ce qu'a écrit Marguerite Duras, on comprend toutes les fines nervures d'un texte particulièrement mystérieux », lit-on dans Le Figaro, corroborant en cela le son de cloche rapporté par une grande partie de la presse. Mais hormis quelques exceptions, l'intérêt médiatique semble se focaliser sur le seul théâtre de Marguerite Duras, dont on sait pourtant qu'elle avait plusieurs cordes à son arc.

\section{Le théâtre selon Marguerite Duras}

La version anglaise de l'encyclopédie en ligne Wikipedia présente Duras comme «a French writer and film director», 
quand son pendant français évoque une «écrivaine, dramaturge, scénariste et réalisatrice française». Pour nous faire une idée plus exacte de l'activité de cet auteur, nous sommes allée examiner les dénominations qui lui sont appliquées dans la base de données de la Bibliothèque nationale de France, réputée pour sa rigueur classificatoire, ce qui nous a valu de découvrir ceci :

Country : France

Language : français

Gender : féminin

Birth : Gia Dinh (Vietnam), 04-04-1914

Death : Paris, 03-03-1996

Note : Romancière, cinéaste et dramaturge

Pseudonyme de Marguerite Donnadieu

Mais ce qui nous intéressait le plus, c'était de découvrir dans quelles catégories était classée l'œuvre de Marguerite Duras déposée à la BNF. Le résultat est présenté par cette institution dans un tableau synthétique qui nous informe, par conséquent, que Marguerite Duras n'était pas seulement «writer and film director», ni même uniquement "écrivaine, dramaturge, scénariste et réalisatrice», mais qu'elle a également composé de la musique, figuré comme actrice dans quatre œuvres, qu'elle a été parolière de deux chansons, traductrice, etc.

\begin{tabular}{|c|c|c|}
\hline $\begin{array}{l}\text { Auteur du texte (269) } \\
\text { Auteur adapté (37) } \\
\text { Réalisateur (28) } \\
\text { Adaptateur (23) } \\
\text { Voix parlée (19) } \\
\text { Metteur en scène (12) } \\
\text { Traducteur (12) }\end{array}$ & $\begin{array}{l}\text { Scénariste (8) } \\
\text { Préfacier (5) } \\
\text { Acteur (4) } \\
\text { Contributeur (3) } \\
\text { Parolier (2) } \\
\text { Auteur de } \\
\text { originale (2) } \\
\text { Annotateur (2) }\end{array}$ & $\begin{array}{l}\text { adaptée (1) } \\
\text { Auteur du commentaire } \\
(1) \\
\text { Interviewer (1) } \\
\text { Auteur ou responsable } \\
\text { intellectuel (2) } \\
\text { Autre (74) }\end{array}$ \\
\hline
\end{tabular}


En d'autres termes, la BNF dispose de 269 textes dont Marguerite Duras est l'auteur, 8 œuvres dont elle est la scénariste, 5 qu'elle a préfacées, 37 adaptées par d'autres auteurs à partir de ses œuvres, 12 qu'elle a mises en scène, etc. Il faut aller dans les différentes rubriques pour connaître le détail des œuvres en question; ainsi par exemple, à la rubrique Auteur du texte. 269 documents, nous avons notamment :

L'Amant (1984), roman

L'Amour (1971), récit

L'Après-midi de Monsieur Andesmas (1962), roman

Un barrage contre le Pacifique (1950), roman

Détruire, dit-elle (1969), roman

Etc.

Ce que nous recherchions dans cette base de données, c'étaient d'éventuels doublons, avec une même œuvre se retrouvant dans deux catégories différentes. C'est notamment le cas d'India Song :

India Song : film (1974)

Long métrage. - D'après la pièce de théâtre de Marguerite Duras India song. - Sortie en France en 1975

India Song est donc un film de 1974, tiré d'une pièce de théâtre.

Dans un texte publié par Le Nouvel Observateur, Odile Quirot présente Marguerite Duras en ces termes:

Aucune romancière n'a aimé les acteurs autant qu'elle. D'où la fureur de Marguerite Duras quand, en 1993, elle apprit que Nathalie Sarraute était enfin jouée par la Comédie-Française, mais pas elle. Il se raconte qu'un soir, Jacques Lassalle, alors administrateur du Français, vit une petite silhouette l'attendre : «C'est moi Duras » - et celle-ci de lui passer un savon.

En 2002, quand Savannah Bay entre au répertoire du Français, dans une mise en scène d'Éric Vigner, elle n'est plus de ce monde. Chez Duras, dès l'origine, roman, théâtre et cinéma 
n'ont toujours fait qu'un. De la page à la scène et à l'écran : elle adapte, recycle, récrit. Les Viaducs de la Seine-et-Oise deviendront L'Amante anglaise, et La Musica deuxième fut d'abord un film.

Si Madeleine Renaud fut son interprète fétiche, Michael Lonsdale, Delphine Seyrig, Suzanne Flon, Catherine Sellers, Bulle Ogier et Sami Frey comptèrent aussi. Le théâtre de Duras est indissociable de ces voix magnifiques. Côté mise en scène, Claude Régy l'accompagna dans les années 1960, puis passa à d'autres découvertes, et Duras monta parfois elle-même ses textes.

Pour toutes ces raisons sans doute, et tant qu'elle fut là, son théâtre, joué, célébré dans le monde entier, ne le fut pas par les grands metteurs en scène qui avaient le souci de réinventer le théâtre. Il faudra attendre 1997 pour que Bob Wilson dirige Michel Piccoli dans La Maladie de la mort, et 2008 pour que Patrice Chéreau s'attache, avec Dominique Blanc, à La Douleur. (Quirot)

Ce qui précède vient conforter l'impression suscitée par l'examen des œuvres de Duras déposées à la BNF, à savoir qu'il pouvait y avoir des allers et retours entre divers genres, d'où le fait, selon Odile Quirot, que, dès l'origine, roman, théâtre et cinéma n'aient fait qu'un : « elle adapte, recycle, réécrit ».

Il est intéressant de confronter l'affirmation d'Odile Quirot sur le caractère populaire du théâtre de Duras avec les confidences de quelqu'un qui a bien connu l'écrivain, le metteur en scène Didier Bezace :

Quand j'ai dit à Marguerite Duras que Le Square était une pièce faite pour un théâtre populaire, elle m'a répliqué : "Mais je n'écris pas de théâtre populaire! » Je dois d'ailleurs avouer que, pendant longtemps, son théâtre m'est apparu comme une sorte de bavardage. Dans les années 1975-1985, il avait été totalement accaparé, avec l'assentiment de Duras, par Madeleine Renaud et Michael Lonsdale. Pour moi, tout cela relevait à l'époque d'un rituel un peu mondain. 
$[\ldots]$

C'est la lecture du Square - une pièce de 1955; Duras avait été exclue du PC [en mars 1950, NDLR] — qui m'a rapproché d'elle. Ce texte est au croisement de l'intime et du politique. Duras m'a dit l'avoir écrit en écoutant les gens se taire dans les squares. Je lui avais demandé les droits quand j'étais au Théâtre de l'Aquarium : "Mon théâtre ne se joue qu'à Paris », m'avait-elle répondu — j'ai toujours la lettre. Et moi : «Mais l'Aquarium est à la Cartoucherie de Vincennes. » Je vous passe le feuilleton de nos conversations téléphoniques. Enfin, elle m'a dit : «D'accord, montez Le Square avec Gérard Depardieu et Bulle Ogier.» Et moi : « Non, à l'Aquarium, on choisit ses distributions. »

\section{$[\ldots]$}

En 1992, je décide de monter ses conversations avec François Mitterrand parues dans L'Autre Journal. Je me dis: si je demande les droits à Marguerite, ça va être l'enfer, elle va vouloir tout récrire. Alors je fonce, je prends un avocat. Duras téléphone tous les jours, je ne réponds pas. La première arrive, Michel Cournot fait un très beau papier dans Le Monde. Deux jours plus tard, Duras est là. Elle tombe amoureuse du spectacle, de mon idée de faire jouer son rôle par une petite fille de 11 ans : "Maintenant, tu montes ce que tu veux », m'a-telle dit. Mais elle n'est plus là pour voir Le Square, que je mettrai finalement en scène en 2003 à... Aubervilliers. (dans Quirot)

"Je n'écris pas de théâtre populaire », déclare Duras à Bezace, ce qui montre bien que les commentateurs, à l'instar d'Odile Quirot, peuvent parfois être en désaccord avec l'auteur. Autre point de vue d'un connaisseur de l'œuvre de Marguerite Duras, celui du comédien Michael Lonsdale :

Q. Dans quelles circonstances avez-vous connu Marguerite Duras?

R. En 1968: les Barrault avaient été chassés de l'Odéon et s'étaient réfugiés dans un petit théâtre, impasse Récamier. Ils montaient L'Amante anglaise, et c'est Claude Régy qui a 
proposé mon nom. Je me souviens que Marguerite était présente aux répétitions. Elle modifiait en permanence le texte. Une pièce était toujours pour elle « $a$ work in progress ». À quelques jours de la représentation, je lui demandais d'arrêter : je ne savais plus quelle était la bonne version à jouer.

\section{$[\ldots]$}

Q. Comment se situait-elle dans la littérature de son temps?

R. Je crois qu'elle n'aimait guère ses contemporains. Je l'ai entendue dire: "Il n'y a que deux écrivains en France: Simenon et moi. » Un jour, nous nous sommes opposés, car elle dénigrait Tchekhov. Cet auteur parle comme personne du mal d'amour entre les êtres. Elle savait bien qu'il la surpassait. Au fil des années, elle s'est mise à avoir un avis tranché sur tout. Je me souviens qu'elle avait interviewé une directrice de prison qu'elle avait violemment attaquée, la mettant en cause, avec brutalité. Elle mélangeait son œuvre et la réalité. Elle s'est intéressée à l'affaire Villemin à cause de L'Amante anglaise [tiré d'un fait divers analogue, NDLR]. Elle est allée voir la maison du drame, a rencontré le juge d'instruction. Mais elle a refusé de voir la mère, sur laquelle elle a pourtant porté un jugement dans un texte célèbre. Elle préférait rêver ses personnages.

Q. N'y a-t-il pas une Marguerite Duras d'avant L'Amant et une d'après?

R. Nous formions avec Marguerite une petite bande très joyeuse, jusqu'au jour où Yann [Yann Andrea, son dernier compagnon, NDLR] est arrivé. Il l'a sauvée, mais il l'a aussi coupée de ses amis. Elle était devenue difficile. Le jour où elle reçut le prix Goncourt, on est allés fêter ça au Théâtre du RondPoint, chez les Barrault. Elle réglait ses comptes avec tout le monde. Elle s'en est prise à une critique présente en lui lançant: "Vous m'avez assassinée. » Elle a vécu assez mal le succès de L'Amant - pas son meilleur livre, car elle écrit au « je ». Elle a d'ailleurs écrit L'Amant de la Chine du Nord pour rectifier le tir après l'adaptation cinématographique de JeanJacques Annaud, qui ne lui plaisait pas. (de Montety) 
«Elle modifiait en permanence le texte; une pièce était toujours pour elle "a work in progress"», reconnaît M. Lonsdale, confirmant en cela le point de vue de Didier Bezace: "si je demande les droits à Marguerite, ça va être l'enfer, elle va vouloir tout récrire ». Tout semble s'être passé comme si Marguerite Duras n'en avait que faire des codes du théâtre ou même du cinéma, des arts qu'elle n'a abordés que de manière fortuite, voire accidentelle, et qu'elle voulait, de toute façon, placer sous la prépondérance de l'écrit, ainsi qu'elle le confie ci-dessous :

Je suis un écrivain, rien d'autre qui vaille la peine d'être retenu. Quand on écrit, si vous voulez, on est cette absence permanente à l'autre qui dure pratiquement le temps que dure la vie, le temps que dure l'écriture. Je ne sais pas jusqu'à quel âge j'écrirai mais je sais que depuis l'âge de dix-huit ans je ne pense qu'à écrire. J'ai eu des accidents de parcours, des enfants, des hommes, mais là où je ne peux pas vous répondre, je ne sais pas si ces accidents ne sont pas eux-mêmes des écrits; c'est un dédoublement; c'est l'équivalent d'un déplacement de soi; j'en parle mal; je ne peux pas en parler bien parce que je pense à l'ambiguïté fondamentale de l'écrit, qui se reporte sur l'écrivain, qui doit être l'ambiguïté fondamentale d'écrire. C'est comme quoi cette personne entière qui voit et qui entend et qui parle a besoin de ne plus parler, d'être aveugle, de se boucher les yeux, de se boucher les oreilles pour retrouver ce qu'elle a vécu, pour l'écrire.

Commentaire de la présentatrice : Un écrivain et rien d'autre. En 1984, interrogée par Marianne A. pour France Culture, Marguerite Duras revendique cet unique qualificatif pour se définir, ajoutant drôlement que tous les accidents de parcours dans lesquels elle classe les enfants, les hommes tout comme sûrement l'alcool, les maladies, les combats politiques n'auront été que des combustibles nécessaires à l'alimentation de cette passion dévorante qui l'aura occupée toute sa vie: écrire. (Duras, 2013) 
«Un écrivain et rien d'autre »: doit-on entendre par là que Duras n'avait aucune considération pour ses autres réalisations, dont les films, par exemple, ou se considérait-elle comme étant un mauvais metteur en scène lorsqu'elle montait certaines de ses pièces au théâtre?

À propos d'une autre de ses œuvres hybrides, La Musica, Duras livre dans une interview les raisons pour lesquelles les deux parties de l'œuvre sont distantes de vingt ans :

M.D. Ce sont des choses qu'on traîne avec soi, très souvent, tous les écrivains. C'est très rare qu'on arrive à les faire. Ce deuxième acte, je vous dis que ça fait vingt ans que j'y pense. J'ai fini quand même par l'écrire.

Q. Et pourtant il y a un ordre dans l'écriture, dans votre écriture, qui n'est jamais le même, finalement.

M.D. J'espère!

Q. Je veux dire le roman, le film, le théâtre, ou alors quelquefois le théâtre, le cinéma, le roman, ou alors le cinéma tout seul, il n'y a jamais d'ordre finalement, et là arrive ce deuxième acte, cette deuxième Musica et également la mise en scène. Qu'est-ce que c'est que la mise en scène, c'est un point d'orgue?

M.D. C'est la mise en littérature. Il y a autant de texte dans La Musica qu'en dehors de La Musica; quand par exemple je donne des indications aux comédiens, toute cette partie n'est pas retenue dans l'écrit, mais elle existe... Qui est une partie dont le roman est exempt, qui n'existe pas, à moins de parler du roman qu'on est en train de faire, on le tuerait. Là par exemple, pour préserver quatre répliques, je peux parler une demiheure, pour les justifier. Je peux parler autour d'elles.

Q. Pourtant vous donnez des indications très très précises dans les pièces que vous écrivez pour la mise en scène. On voit vraiment évoluer le personnage, ce qu'il fait, comment il réagit, comment il se retrouve, etc. Et ça, vous pensez à ce moment-là à vous, c'est-àdire à la mise en scène que vous pourrez faire, vous, ou vous pensez à la mise en scène qu'on pourra faire de votre pièce? 
M.D. Je ne pense qu'à moi, toujours. Il n'y a que moi qui mets en scène mes pièces. Quand ça arrive à d'autres, des erreurs qu'ils font (phrase inachevée)... La Musica était plus, jusqu'ici, une situation; c'est pas une pièce de personnages; c'est une situation, un état, de l'amour qui est décrit. Un amour qui se défait, alors qu'il existe toujours, qui apparemment se défait. C'est une situation banale. Avec le deuxième acte, il me semble que j'introduis ce que vous appelez des personnages, c'est-àdire des gens avec certains traits de caractère.

Q. Qu'est-ce que ça vous procure, ce théâtre, cette mise en scène? C'est un jeu pour vous?

M.D. Ça m'intéresse. C'est trop long pour le théâtre. Ça m'intéresse quand même. Ça me force à travailler en profondeur. Si vous voulez, le travail de creusement, d'arrachement que l'on fait quand on fait des textes de livres, des textes qui ne sont pas parlés, je connais ce travail-là pendant la mise en scène, pas pendant l'écriture du texte. L'écriture du texte à proprement parler, ça procède aussi d'un escamotage de quelque chose, de tout l'entour de l'écrit.

Q. Et quand vous l'entendez, c'est toujours bien?

M.D. Mais non, pas toujours.

Q. Vous vous dites c'est bien, c'est bien pour vous?

M.D. Quelquefois je suis très contente. Quelquefois je jette tout. En général, je recommence tout. Dès que je mets en scène. Ce n'est plus du tout la même Musica qu'il y a trois semaines que je répète maintenant. C'est une autre. Mais je pense que celle-là, quand même, à trois semaines de jouer, je pense que celle-là va rester. Mais si on me laissait trois mois, c'est sûr qu'elle disparaîtrait encore.

Q. Est-ce que vous éprouvez souvent cette sensation que l'histoire n'est jamais finie?

M.D. Toujours. [...] de la vie! (Gautier, 1985a) 
«La mise en scène c'est la mise en littérature »: Duras donne l'impression de ne savoir rien faire d'autre, ce qu'elle aurait fortement contredit si on le lui avait suggéré. Sinon, d'ailleurs, pourquoi faire du cinéma ou du théâtre, qui ne sont pas forcément de la littérature, dans la mesure où l'on peut supprimer les dialogues et ne retenir que la gestuelle! Mais l'interview qui précède recèle un autre intérêt, lorsque Duras déclare: "Je ne pense qu'à moi, toujours. Il n'y a que moi qui mets en scène mes pièces. » Bien évidemment, Duras n'a pas été la seule à mettre en scène ses pièces, et elle le sait. Rappelons simplement le souvenir rapporté par M. Lonsdale: «les Barrault avaient été chassés de l'Odéon et s'étaient réfugiés dans un petit théâtre, impasse Récamier. Ils montaient L'Amante anglaise, et c'est Claude Régy qui a proposé mon nom. Je me souviens que Marguerite était présente aux répétitions. » Duras a, donc, au moins une fois, assisté à la mise en scène d'une de ses pièces par quelqu'un d'autre! Autre détail intéressant: lors de la toute dernière interview, reproduite en fin de volume, Duras déclare ceci : «je mets très peu en scène au théâtre ». Il faut donc comprendre que l'écrivaine se considère comme la seule capable de mettre en scène ses pièces.

Dans l'extrait d'interview qui suit, nous pouvons raisonnablement nous interroger sur le sens à donner à la réplique suivante: "India Song c'est pas une pièce, c'est un roman. »

Q. Il y a nombre d'acteurs qui reviennent régulièrement pour interpréter vos personnages.

M.D. On peut dire oui, on peut le dire. 
Q. Est-ce que ça vous donne la sensation que ce que vous avez écrit vous échappe moins?

M.D. C'est moins de travail quand ils me connaissent. Il y a une syntaxe de moi qu'ils connaissent. C'est Sellers qui disait: "Quand Duras dit: "Rien", moi je sais ce que ça veut dire; je sais comment il faut le prendre. »

Q. Comment on accepte la séparation quand on écrit entre ces personnages et ces personnages?

M.D. Vous voulez dire les personnages du théâtre et les personnages du roman? C'est pareil, je les entends de la même façon quand je fais le dialogue. Vous n'en sortirez jamais de ça. Je ne fais pas de différence. India Song c'est pas une pièce, c'est un roman. Vous avez entendu comme ils parlent? Comme sur la scène du Français [cf. la Comédie-Française, n.d.l.r.] (pause, sourire en coin)... les bons jours! (Gautier, 1985b)

« Je ne fais pas de différence. India Song c'est pas une pièce, c'est un roman », voire c'est aussi un film : c'est probablement cette volonté de tout confondre que Marguerite Duras appelait une « mise en littérature ».

La dernière interview que nous avons retenue est intéressante à plus d'un titre. C'est l'animateur José Artur qui interroge Marguerite Duras à la télévision. Il y est question du rapport de l'écrivain avec le public ainsi que de ce fameux mélange de genres évoqué plus haut.

Q. Je vous l'ai déjà dit et je le redis, vous êtes quelqu'un d'assez étonnant, parce que sans faire aucune concession, jamais, à quoi que ce soit, vous avez réussi votre vie, publique - on ne va pas parler de la vie privée, ça vous regarde - mais disons qu'en ne voulant pas plaire au plus grand nombre, finalement, ça vient tout doucement. Alors, vous avez toujours les fidèles, qui adorent ce que vous faites, et puis maintenant le grand public commence à savoir qui est M.D.; ça devient presque une expression: on dit c'est un Duras comme on disait à un moment: "C'est un Kafka, c'est un Beckett.» Alors ça veut 
dire : «C'est peut-être un peu difficile d'accès, mais c'est bien beau. » Est-ce que ça, ça vous fait sourire ou pas?

M.D. Je voudrais l'ignorer ça. Il y a deux temps, vous savez, quand on fait quelque chose. Il y a le temps de le faire, qui est un temps inaliénable..., on est complètement seule dans cette période-là. Et ensuite quand le public s'en empare, quand la chose est livrée au public, c'est un temps qui ne devrait pas regarder l'auteur. Je rêve d'arriver vraiment à me ficher complètement de mes livres et de mes films une fois qu'ils sont sortis, une fois qu'ils sont faits. Mais je pense que je vais y arriver.

Q. Et quand ils sont sur scène, est-ce que vous vous y intéressez encore? Par exemple en ce moment, au Mouffetard, il y a Musica, et il y a Les Eaux et forêts...

M.D. Là oui, ça m'est très proche; ce sont des pièces auxquelles je tiens beaucoup et que j'ai mis [sic] en scène; comme je mets très peu en scène au théâtre, ce sont des pièces qui me sont très très chères...

Q. Musica, je crois, spécialement, est une pièce qui arrive à vous faire pleurer quand vous allez voir le spectacle. Sincèrement, vous avez des larmes dans les yeux.

M.D. Je crois que ça concerne tout le monde, Musica. C'est ce couple qui se défait, qui divorce..., et qui se voit après le divorce, pour la dernière fois, dans un hôtel de la ville... C'est que Musica est un film réaliste.

Q. Ouais...

M.D. Les Eaux et forêts c'est..., un film! Pardon, c'est une pièce de théâtre!

Q. Non, mais c'est un lapsus que j'avais laissé passer volontairement!

M. D. Les Eaux et forêts c'est beaucoup plus abstrait, et j'avoue, enfin, pour moi c'est d'un comique irrésistible...

Q. [...] Alors vous écrivez directement pour le théâtre et directement pour le cinéma, c'est ce que vous souhaitez faire de plus en plus, ne plus mélanger les genres, c'est-à-dire ne plus 
adapter des choses faites pour le roman en un film ou une pièce de théâtre; vous préférez directement créer pour le véhicule du cinéma ou du théâtre.

M.D. Oui, c'est-à-dire que j'aimerais arriver à faire des films en six jours. Non pas pour aller vite, mais parce que je crois qu'il y a un élan, il y a un élan du comédien et de la mise en scène, il y a une dynamique, une dynamique du film qui, au bout d'une semaine, se perd... C'est une sorte d'instantanéité, je n'aime pas beaucoup ces mots-là... Je voudrais arriver à faire des films en six jours; j'ai deux projets comme ça.

Q. Combien de temps vous avez mis pour India Song par exemple?

M.D. India Song, j'ai mis quatorze jours... Des journées entières dans les arbres, j'ai mis dix jours plus une demi-journée... (Artur)

Ce que nous pouvons tirer de cet échange, c'est que Marguerite Duras jouissait déjà d'une certaine notoriété, elle qui passait pour un écrivain hermétique, ainsi que le rappelle l'intervieweur: nous sommes en 1976 et le premier grand succès n'interviendra qu'en 1984 avec le Prix Goncourt décerné à L'Amant.

Et puis, il y a ce lapsus, que José Artur dit avoir laissé passer volontairement : "Les Eaux et forêts c'est un film ». Mais faut-il vraiment s'en étonner? La suite est assez intéressante, l'intervieweur évoquant une spécialisation dans l'écriture: pour le théâtre, pour le cinéma, de manière à «ne plus mélanger les genres, c'est-à-dire ne plus adapter des choses faites pour le roman en un film ou une pièce de théâtre ». Bien évidemment, cette interrogation, que l'intéressée ne conteste pas, nous informe incidemment sur le fait que Duras a auparavant copieusement mélangé les genres! Et là, on croyait la réponse évidente et limpide; en fait, Duras essaie encore de 
brouiller les pistes et, dans la réponse qu'elle fait sur le mélange des genres, on voit bien qu'elle n'est toujours pas sortie du cinéma, alors même qu'il était question de théâtre (Les Eaux et forêts) : «Oui, c'est-à-dire que j'aimerais arriver à faire des films en six jours.»

Finalement, c'est à l'un de ses principaux biographes que nous emprunterons la conclusion de cet article, qui résume très bien les circonstances, souvent fortuites, qui ont présidé à l'émergence de telle ou telle œuvre de Duras, dont la méthode se retrouve entièrement dans cette confidence : "J'ai écrit une pièce de théâtre sans le savoir. »

On pourrait, en simplifiant un peu les choses, dire que Marguerite Duras est «tombée » par hasard dans le théâtre. En 1956, le metteur en scène Claude Martin lui suggère d'adapter pour lui Le Square, le roman qu'elle a publié chez Gallimard l'année précédente. Chose facile, car le roman en question consiste en un long dialogue entre deux personnages: une bonne à tout faire et un voyageur de commerce devisant dans un jardin public. Les deux personnages, au début, ne se connaissent pas. Nous ne les connaissons pas davantage. Ils ne se connaîtront pas beaucoup plus à la fin de cette rencontre fortuite, mais, en revanche, nous en saurons assez sur eux pour ne plus les oublier. Le Square, créé au Studio des ChampsElysées au début de la saison 1956-1957, deviendra au cours des années l'une des pièces les plus jouées de son auteur. Les articles de presse qui accompagnent la création sont révélateurs: "Une pièce involontaire", titre L'Express du 14 septembre 1956; "Un roman de Marguerite Duras conçu comme une pièce ", lui répond Franc-Tireur le même jour. Ellemême confie à L'Information le 15 septembre : "J'ai écrit une pièce de théâtre sans le savoir », tandis que Demain, publiant l'interview qu'elle a accordée pour la circonstance, titre : « Du théâtre malgré moi». En fait, Marguerite Duras était prédestinée à écrire pour le théâtre, tant son sens du dialogue est évident dès la parution du Barrage contre le Pacifique lequel ne manquera pas d'être à son tour adapté pour la scène 
un peu plus tard par Geneviève Serreau, avec la complicité de la romancière. Ajoutons à cela une rencontre miraculeuse, celle d'une des plus grandes actrices du siècle, la sublime Madeleine Renaud qui ne créera pas moins de quatre pièces majeures écrites tout exprès pour elle. Elle deviendra une de ses meilleures amies et portera à son plus haut point de perfection la petite musique durassienne, cette Musica, titre d'une de ses pièces les plus attachantes, qui, paradoxalement pour un art qui doit presque tout à la parole, est le plus souvent chez Marguerite Duras une musique intérieure. (Vallier, p. 138) 


\section{Bibliographie}

ARTUR, José. (1976), « Duras explique pourquoi elle n'aime pas trop les interviews », 12 décembre, <http://www.ina.fr/video/I04258929/marguerite-durasinterviewee-par-jose-artur-video.html>.

BelHAOUES, Linda et Élise LuCET. (2014), « La passion de Fanny Ardant pour Marguerite Duras », FranceTVinfo, 20 janvier 3 février, <http://culturebox.francetvinfo.fr/scenes/ theatre/interview-la-passion-de-fanny-ardant-pourmarguerite-duras-148233>.

DURAS, Marguerite. (2013), "La passion suspendue : entretiens inédits avec une Marguerite Duras mise à nu», archive diffusée dans le cadre de l'émission L'Humeur Vagabonde, France Inter, 21 janvier, $<$ http://culturebox.francetvinfo.fr/livres/essaisdocuments/la-passion-suspendue-entretiens-inedits-avecune-marguerite-duras-mise-a-nu>.

GANDILLOT, Sarah. (2014), «Marguerite Duras célébrée au théâtre à Paris », 20 Minutes, 21 janvier.

GAUTIER, Marie-Claire. (1985a), «La Musica 2, écrite vingt ans après le premier acte », 1er mars, <http://www.ina.fr/video/PAC00020449>.

-. (1985b), "Sur la mise en scène, la non séparation [sic] qu'elle [Duras] fait entre le théâtre et le roman », 2 mars, $<$ http://www.ina.fr/video/PAC00020459/margueriteduras-video.html $>$. 
HÉLIOT, Armelle. (2014), "Didier Bezace, metteur en scène et interprète de Marguerite Duras », Le Figaro, 12 février, $<$ http://blog.lefigaro.fr/theatre/2014/02/didier-bezacemetteur-en-scene.html>.

MonTETy, Étienne de. (2011), «Michael Lonsdale raconte Marguerite Duras », Le Figaro - Livres, <http://www.lefigaro.fr/livres/2011/10/25/0300520111025ARTFIG00705-michael-lonsdale-racontemarguerite-duras.php>.

Plantin, Marie. (2014). " Une année sous le signe de Marguerite Duras », Première, <http://spectacles.premiere.fr/NewsPhotos/2014-une-annee-sous-le-signe-de-MargueriteDuras-3945391>.

QuiroT, Odile. (2014), « Duras dure toujours : c'est du théâtre populaire», Le Nouvel Observateur, 8 février, $<$ http://bibliobs.nouvelobs.com/actualites/20140207.0BS5 547/marguerite-duras-dure-toujours-c-est-du-theatrepopulaire.html>.

VALLIER, Jean. (2006), Marguerite Duras, la vie comme un roman, Paris, Éditions Textuel. 


\section{Résumé}

"J'ai écrit une pièce de théâtre sans le savoir. » Ainsi s'exprimait Marguerite Duras à propos du Square, sa première pièce de théâtre, adaptée sur commande d'après le roman éponyme. On sait que Duras a souvent recyclé ses textes, que l'on a retrouvés sous la forme de nouvelles, de récits, de romans, de pièces de théâtre, de films, pour ne citer que La Musica ou India Song. Duras a même écrit des chansons. C'est ainsi qu'on s'étonne à peine qu'une artiste aussi versatile ait dû, au cours d'une interview, se reprendre après un lapsus: "Les Eaux et forêts c'est un film! Pardon, c'est une pièce de théâtre! » En cette année 2014, qui marque le centenaire de sa naissance, et alors que tant d'artistes adulés de leur vivant sombrent dans l'anonymat sitôt morts, nous avons voulu savoir ce qu'il restait de Marguerite Duras dans la mémoire des Français près de vingt ans après sa mort.

\section{Abstract}

"I wrote a play without knowing it." So said Marguerite Duras about The Square, her first play, adapted to order according to the eponymous novel. We know that Duras often recycled her texts, which could come out as news, stories, novels, plays, films, just to quote La Musica or India Song. Duras had even written songs. Thus, it is hardly surprising that such a versatile artist had in an interview to recover from a slip of the tongue: "Les Eaux et forêts is a film! Forgiveness, it is a play!" This year 2014 marking the centenary of her birth, and after so many adulated artists in their lifetime have sunk into anonymity right after their death, we wanted to know what was left of Marguerite Duras in the memory of French people almost twenty years after she passed away. 